\title{
Preocupação com a imagem corporal em discentes do curso de Educação Física
}

\author{
Concern about body image in students of the \\ Physical Education Course
}

Otacélio Galber Mota Sousa, ${ }^{1}$ Romário Pinheiro Lustosa, ${ }^{2}$ Rosane de Almeida Andrade, ${ }^{3}$ Natália Macedo Uchoa, ${ }^{4}$ Thiago Medeiros da Costa Daniele, ${ }^{5}$ Francisco Nataniel Macedo Uchoa ${ }^{3}$

${ }^{1}$ Faculdade da Grande Fortaleza (FGF), Fortaleza, CE, Brasil.

¿2entro Universitário Estácio do Ceará, Fortaleza, CE, Brasil.

3Universidade Trás dos Montes e Alto Douro (UTAD), Vila Real, Portugal.

${ }^{4}$ Universidade de Fortaleza (UNIFOR), Fortaleza, CE, Brasil.

EUniversidade Federal do Ceará (UFC), Fortaleza, CE, Brasil.

Recebido em: Fevereiro 2016 / Aceito em: Março 2016

galbersousa@hotmail.com

\section{RESUMO}

A imagem corporal (IC) é a imagem que o indivíduo reproduz em sua mente, podendo variar por fatores psicológicos, dependendo do nível de preocupação com partes do corpo ou com o seu todo. A preocupação excessiva com a aparência poderá gerar distúrbios emocionais e incentivar a práticas prejudiciais à saúde para o alcance do corpo desejado. Objetivo: o objetivo dessa pesquisa foi investigar a satisfação com a IC em discentes do curso de licenciatura em Educação Física de uma instituição de ensino privada de Fortaleza - CE/ Brasil. Método: trata se de uma pesquisa quantitativa, transversal e descritiva. Analisando o nível de preocupação com imagem corporal de 100 universitários, por meio da aplicação do questionário validado BSO (Body Shape Questionare), que é composto por 34 questões, as quais qualificam os entrevistados em nenhum, leve, moderada e grave preocupação com o próprio corpo. A tabulação de dados foi realizada no Microsoft Excel 2010. Resultados: os resultados mostraram que a maioria dos entrevistados (74\%) não tem nenhuma preocupação com sua atual forma física, demonstrando estarem altamente satisfeitos com o seu corpo. O público feminino demonstrou maior preocupação com a IC do que os homens, mesmo assim a maioria (60\%) foi qualificada com nenhuma preocupação com a IC. Considerações finais: ao final dessa pesquisa, podemos concluir que a maioria dos universitários do curso de
Educação Física está satisfeitos com o seu corpo, por não demonstrarem preocupação excessiva com a sua autoimagem, segundo resultado do BSQ.

Palavras-chave: Imagem Corporal; Universitários; Educação Física.

\section{ABSTRACT}

Body image $(B I)$ is the image that the individual plays in your mind, and may vary by psychological factors depending on the level of concern with body parts or the whole. Excessive concern with appearance could lead to emotional disturbances and encourage prejudicial practices to health in order to achieve the desired body. Objective: the aim of this survey was to investigate satisfaction with $\mathrm{Bl}$ in students from a Physical Education course of a private education institution in Fortaleza - CE / Brazil. Method: this is a quantitative, transversal and descriptive survey. We analyze the level of concern about $B /$ of 100 college students, through the validated questionnaire BSO (Body Shape Questionare), composed of 34 questions, which qualify the respondents in none, mild, moderate and severe concern with their own body. The tabulation of data was performed in Microsoft Excel 2010. Results: the results showed that the majority of respondents (74\%) have no concern with your current fitness, demonstrating to be highly satisfied with their body. The female audience showed 
to be more concern with $B /$ than men, yet the majority (60\%) was qualified with no concern for the Bl. Closing remarks: at the end of this research we can conclude that most students of the Physical Education course are satisfied with your body, showing no excessive concern with their self-image, as a result of the BSO.

Keywords: Body Image; College Students; Physical Education.

\section{INTRODUÇÃO}

A imagem corporal (IC) foi definida como a figuração de nosso corpo formada em nossa mente. ${ }^{1} \mathrm{O}$ interesse em pesquisar esse tema vem crescendo nos últimos anos; nota-se que grande parte da população está insatisfeita com sua imagem corporal. ${ }^{2-5}$

A insatisfação corporal pode ser definida como a disparidade entre o tamanho percebido pela pessoa e o tamanho que ela gostaria de ter realmente. ${ }^{7}$ Estudos têm encontrado predomínio relativamente elevado de insatisfação corporal no público feminino. ${ }^{8-11}$

A mídia é o principal agente responsável no descontentamento da imagem corporal de jovens. A exposição de corpos de modelos/atrizes costuma causar impacto imediato na idealização da imagem sociocultural em sujeitos do sexo feminino. ${ }^{3,12,13}$ Schilder já afirmava em seus estudos que existe um intercâmbio contínuo entre a nossa imagem corporal e a dos outros. ${ }^{14}$

Ademais, existem evidências de que o estado nutricional, a falta de atividade física e a composição corporal, como, sobrepeso e obesidade estão vinculados com a insatisfação da IC..$^{10,15} \mathrm{O}$ comportamento alimentar inadequado é frequente em universitários que apresentam relação conturbada com o alimento e com o corpo, podendo ter consequências físicas e psíquicas. ${ }^{16}$ O ingresso na vida universitária provoca alterações no comportamento alimentar, ${ }^{17}$ e a exposição ao novo meio social pode trazer como consequência aumento da insatisfação corporal. Estudos têm sido realizados no sentido de avaliar a insatisfação com o corpo e também as atitudes alimentares inadequadas em universitários. ${ }^{18,19}$

Pesquisas apontam que a insatisfação com a IC é mais percebida entre o final da adolescência e início da fase adulta, período que os jovens ingressam no ensino superior. Sabendo desses dados, universitários podem constituir um grupo de risco para que possam contrair distúrbios emocionais por se preocuparem excessivamente com sua aparência. ${ }^{5}$

O período recém-ingresso na universidade, onde o indivíduo adquire novas amizades e obrigações acadêmicas, pode levar o acadêmico a costumes pouco saudáveis, podendo afetar em sua imagem corporal, tornando assim relevante as pesquisas sobre a percepção corporal com o público universitário. ${ }^{20}$

Seguindo essa linha de pensamento, a motivação para a realização do estudo é: o aluno de Educação Física está satisfeito com sua imagem corporal? Por esta razão, o objetivo do estudo é investigar a preocupação com a imagem corporal de alunos do curso de licenciatura em Educação Física, mais precisamente analisar o nível de satisfação com sua autoimagem e comparar por sexo.

\section{MÉTODO}

O trabalho foi realizado por meio de uma pesquisa quantitativa, transversal e descritiva. A amostra foi constituída por 100 alunos de uma rede privada de ensino, discentes do curso de Licenciatura em Educação Física na cidade de Fortaleza - Ceara/Brasil. Foi aplicado o questionário ao longo do mês de outubro de 2015.

Foram incluídos na pesquisa aqueles que, por meio da assinatura do Termo de Consentimento Livre e Esclarecido, aceitaram participar da pesquisa voluntariamente. A exclusão foi feita por meio da não voluntariedade em participar do estudo.

Como instrumento de pesquisa, foi utilizado o questionário Body Shape Questionnaire (BSO) que dispõe de 34 perguntas de autopreenchimento, com respostas variadas de 1 (nunca) a 6 (sempre), de acordo com o modo de escala em Likert de pontos. O BSO se subdivide em 4 sub escalas que analisam a auto percepção da forma corporal (22), preocupação comparativa (5), atitude (5) e por fim alterações graves (2). Assim, o BSQ avalia a insatisfação geral da imagem corporal, por meio do nível de preocupação com a autoimagem.

Quanto maior a pontuação nessa escala, maior será a insatisfação com a autoimagem, devido a maior preocupação com o formato do corpo. O escore para análise do resultado é obtido por meio da soma das 34 questões, podendo variar de 34 a 204 pontos. Os escores obtidos classificam o teor de preocupação com a aparência física, em 4 níveis a saber: nenhuma preocupação com a imagem corporal (soma menor ou igual a 80), leve preocupação com a imagem corporal (soma maior que 80 e menor que 111), moderada preocupação com a imagem corporal (soma maior que 110 e menos que 141) e grave preocupação com a imagem corporal (soma maior que 140).

Os dados foram analisados a partir de médias aritméticas e percentuais extraídos dos resultados obtidos dos questionários, com o auxílio do programa Microsoft Office Excel 2010.

\section{RESULTADOS E DISCUSSÃO}

Na tabela 1 temos os resultados encontrados do público em geral da pesquisa. Dos 100 indivíduos, 70\% são do sexo masculino. A idade média dos pesquisados é de 27,17 anos. O BSO médio do público analisados foi de 70,13 pontos. Do total dos participantes (74\%) tiveram um resultado no BSO abaixo de 80 pontos, que mostram que não tem nenhuma preocupação com a autoimagem, refletindo grande satisfação com seu corpo; $23 \%$ dos sujeitos somam leve a moderada preocupação com a autoimagem. Apenas (3\%) dos entrevistados tiveram seu resultado com soma maior que 140 , que demonstra grave preocupação com a autoimagem, ou seja, esses indivíduos são extremamente insatisfeitos com sua percepção corporal.

Os resultados encontrados nesta tabela, no quesito satisfeito com a autoimagem, são totalmente diferentes dos encontrados por outros estudos que relatam que um número considerável de universitários avaliados demonstraram alguma insatisfação com a IC. ${ }^{5,21}$ 
Tabela 1 - Dados gerais do estudo.

$\begin{array}{lc}\text { Sexo M / F (\%) } & 70 \% / 30 \% \\ \text { Idade Média ( } \pm \text { DP) } & 27,17( \pm 7,03) \\ \text { BSO Média ( } \pm \text { DP) } & 70,13( \pm 25,05) \\ \text { Nenhuma preocupação com a autoimagem } & 74 \% \\ \text { Leve preocupação com a autoimagem } & 19 \% \\ \text { Moderada preocupação com a autoimagem } & 4 \% \\ \text { Grave preocupação com a autoimagem } & 3 \%\end{array}$

$\mathrm{Na}$ tabela 2, encontramos os resultados estratificados por sexo. Apenas duas $(6,66 \%)$ das mulheres obtiveram pontuação acima de 140 pontos, que revela grave preocupação com a autoimagem, ou seja, muito insatisfeitas com o corpo. Já no sexo masculino $80 \%$ indicam nenhuma preocupação com a aparência, ou seja, estão muito satisfeitos com o próprio corpo. Os resultados da análise pelo gênero feminino têm semeIhança com uma pesquisa que também utilizou o BSO na sua análise, mostrando que o sexo feminino tem tendência a ter maior preocupação com a forma física em relação à média do BSO do público geral. ${ }^{11}$

Em outra pesquisa realizada com universitários, no que tange ao sexo, as mulheres apresentaram maior insatisfação com imagem corporal. Na ocasião $46 \%$ das mulheres relataram que estão pouco satisfeitas com o próprio corpo. ${ }^{22}$

Concordando com uma análise feita com 1230 indivíduos, demonstrando que pessoas do sexo masculino tem (61\%) menos chances de serem insatisfeitos com o próprio corpo. Nesta pesquisa o BSO médio das mulheres $(82,33)$ foi quase $(26,85 \%)$ maior que o BSO médio dos homens $(64,9)$ e $(17,40 \%)$ maior que o BSQ médio geral $(70,13)$, evidenciando a maior preocupação com a IC nesse estudo em mulheres. ${ }^{23}$

Num adicional estudo, aponta que o sexo masculino tem uma maior aceitação do corpo, apesar de relatarem algum tipo de incomodo quanto a sua aparência. No estudo ainda pode-se notar que os homens desejam aumentar a massa muscular enquanto que mulheres preferem manter suas medidas menores. ${ }^{24}$

Em recente revisão literária notou-se que a quantidade de artigos que concluíam qual o público mais acometido por insatisfação corpórea, os resultados equipararam-se, mostrando que o número de homens incomodados com sua IC está aumentando. ${ }^{25}$

\section{CONSIDERAÇÕES FINAIS}

Foi constatada pequena preocupação com a insatisfação acentuada com a IC dos sujeitos do estudo. Apesar dos universitários terem tendências a ter insatisfação com a IC, a hipótese não foi confirmada em discentes do curso de Educação Física da referida instituição, mostrando de uma forma geral que os alunos estão muito satisfeitos com o seu corpo atual.

O público feminino demonstrou maior preocupação com a forma física do que o público masculino; mesmo assim, a maioria teve resultados que identificaram satisfação na atual imagem do seu corpo.

Sugere-se replicar o estudo com outros cursos. Assim poderíamos identificar se cursos de outras áreas tendem a ter maiores insatisfações com o corpo devido a não terem instruções ou práticas em atividades físicas.

\section{REFERÊNCIAS}

1. Schilder, P. A Imagem do Corpo: as energias construtivas da psique.São Paulo: Martins Fontes; 1994.

2. Kakeshita I. Almeida S. Relação entre índice de massa corporal e a percepção da auto-imagem em universitários. Rev Saude Publica 2006;40(3):497-504.

3. Rodgers R, Chabrol H, Paxton S. An exploration of the tripartite influence model of body dissatisfaction and disordered eating among Australian and French college women. Body Image 2011;8(3):208-215. doi: 10.1016/j.bodyim.2011.04.009.

4. Flament MF, Hill E, Buchholz A, Henderson K, Tasca G, Goldfield G. Internalization of the thin and muscular body ideal and disordered eating in adolescence: The mediation effects of body esteem. Body Image 2012;9(1):68-75. doi: 10.1016/j.bodyim.2011.07.007.

5. Silva DAS, Pereira IMM, Oliveira AC. Cabral de. Impacto da escolaridade materna e paterna na perceção da imagem corporal em acadêmicos de Educação Física. Motri 2012;(2):2231. doi: 10.6063/motricidade.8(2).709.

6. Martins CR, Petroski EL. Insatisfação com a imagem corporal em adolescentes do sexo feminino de uma cidade de pequeno porte: prevalência e correlações. Motricidade 2015;11(2):94106. doi: $10.6063 /$ motricidade.3670.

7. Gardner RM, Brown DL. Body image assessment: A review of figural drawing scales. Personality and Individual Differences 2010;48(2):107-111. doi: 10.1016/j.paid.2009.08.017

8. Alves E, Vasconcelos F, Calvo M, Neves J. Prevalência de

Tabela 2 - Dados da Imagem Corporal estratificados por sexo.

\begin{tabular}{|c|c|c|}
\hline & Masculino & Feminino \\
\hline Quantidade da Amostra & 70 & 30 \\
\hline Idade Média ( $\pm \mathrm{DP}$ ) & $27,43( \pm 6,13)$ & $26,57( \pm 8,87)$ \\
\hline BSO Média ( $\pm \mathrm{DP})$ & $64,9( \pm 22,76)$ & $82,33( \pm 26,28)$ \\
\hline Nenhuma preocupação com a autoimagem & $80 \%$ & $60 \%$ \\
\hline Leve preocupação com a autoimagem & $15,71 \%$ & $26,66 \%$ \\
\hline Moderada preocupação com a autoimagem & $2,85 \%$ & $6,66 \%$ \\
\hline Grave preocupação com a autoimagem & $1,42 \%$ & $6,66 \%$ \\
\hline
\end{tabular}


sintomas de anorexia nervosa e insatisfação com a imagem corporal em adolescentes do sexo feminino do Município de Florianópolis, Santa Catarina, Brasil. Cad. Saúde Pública, Rio de Janeiro 2008;24(3):503-512. doi: 10.1590/s0102 $311 \times 2008000300004$.

9. Martins CR, Pelegrini A, Matheus SC, Petroski EL. Insatisfação com a imagem corporal e relação com estado nutricional, adiposidade corporal e sintomas de anorexia e bulimia em adolescentes. Revista de Psiquiatria do Rio Grande do Su 2010;32(1):19-23.

10. Miranda V, Conti M, Bastos R, Ferreira M. Insatisfação corporal em adolescentes brasileiros de municípios de pequeno porte de Minas Gerais. Jornal Brasileiro de Psiquiatria 2011;60(3): 190-197. doi: 10.1590/s0047-20852011000300007.

11. Fortes LS, Cipriani F, Coelho F, Paes S, Ferreira M. Does selfesteen affect body dissatisfaction levels in female adolescents? Rev Paul Pediatria (EnglishEdition) 2014;32(3),236-240. doi 10.1016/s2359-3482(15)30016-6.

12. Amaral ACS, Cordás TA, Conti MA, Ferreira MEC. Equivalência semântica e avaliação da consistência interna da versão em português do sociocultural attitudes Towards Appearance Questionnaire-3 (SATAQ-3). Cad Saúde Pública, Rio de Janeiro 2011;27(8):1487-1497.

13. Gondoli DM, Corning A, Blodgett Salafia E, Bucchianeri M, Fitzsimmons E. Heterosocial involvement, peer pressure for thinness, and body dissatisfaction among young adolescent girls. Body Image 2011;8(2):143-148. doi: 10.1016/j. bodyim.2010.12.005.

14. Schilder P. A imagem do corpo. As energias construtivas da psique. 3. ed. São Paulo: Martins Fontes, 1999.

15. Scherer F, Martins C, Pelegrini A, Matheus S, Petroski E. Imagem corporal em adolescentes: associação com a maturação sexual e sintomas de transtornos alimentares. J Bras Psiquiatr 2010;59(3):198-202. doi: 10.1590/s004720852010000300005

16. Alvarenga MS, Scagliusi FB, Philippi ST. Comportamento de risco para transtorno alimentar em universitárias brasileiras.
Rev Psiq Clín 2011;38(1):3-7.

17. Petribú MMV, Cabral PC, Arruda IKG. Estado nutricional, consumo Alimentar e Risco cardiovascular: um Estudo em Universitários. Rev. Nutr. 2009; 22 (6): 837-846. DOI: http:// dx.doi.org/10.1590/S1415-52732009000600005.

18. Miranda VPN, Filgueiras JF, Neves CM, Teixeira PC, Ferreira MEC. Insatisfação corporal em universitários de diferentes áreas de conhecimento. J Bras Psiquiatr 2012;61(1):25-32.

19. Bosi MLM, Uchimura KY, LuizRR. Eating behavior and body image among psychology students. J Bras Psiquiatr 2009;58(3):1505. doi: 10.1590/S0047-20852009000300002.

20. Ferrari EP, Gordia AP, Martins CR, Silva DA, Quadros TM, Petroski EL. Insatisfação com a imagem corporal e relação com o nível de atividade física e estado nutricional em universitários. Motricidade 2012;8(3):52-58. doi: 10.6063/ motricidade.8(3).1156.

21. Coqueiro RS, Petroski EL, Pelegrini A, Barbosa AR. Insatisfação com a imagem corporal: avaliação comparativa da associação com o estado nutricional em universitários. Revista psiquiatria, Rio Grande do Sul 2008;30(1):31-168.

22. Ferreira LRBA, Dantas SFIM. Nível de satisfação com a imagem corporal entre estudantes e professores do instituto de ciências da saúde da Faculdade Alfredo Nasser -FAN. In: Pesquisar da Faculdade Alfredo Nasser, 3, 2014, Goiânia. Anais eletrônicos. Goiânia: FAN.

23. Rechh RR, Chaves JC, Zanotti LR, Halpern R. Características antropométricas em escolares com insatisfação com a imagem corporal. Coleção Pesquisa em Educação Física, Várzea Paulista 2014;13(1):49-58.

24. Mantovani B, Pinha G, Corrêa B, Bueno L, Freitas M, Damaceno $H$, Hirai R. Nível de satisfação com a imagem corporal de universitários. EFDeportes.com - Revista Digital, Buenos Aires 2009;14(132).

25. Uchôa FNM, Lustosa RP, Rocha MTL, Daniele TMC, Aranha ACM. Causas e implicações da imagem corporal em adolescentes: uma revisão literária. Cinergis 2015;16(4):246252. doi: 10.17058/cinergis.v16i4.6936. 